\title{
Can the increase of bone mineral density following bisphosphonates treatments be explained by biomechanical considerations?
}

\author{
Dominique P. Pioletti ${ }^{a, *}$, Lalao R. Rakotomanana ${ }^{b}$ \\ a Bone Bioengineering Group, STI/BIO-E/CRO, Center for Orthopedic Research, Bat. AAB, EPFL, Swiss Federal Institute of Technology, \\ Lausanne 1015, Switzerland \\ b IRMAR, University of Rennes I, France
}

Received 26 November 2002; accepted 10 October 2003

\begin{abstract}
Objective. We hypothesized that bone mineral density increase following bisphosphonates treatments may be explained by the influence of the drug on the mechanical bone remodeling parameters.

Background. Patients treated with bisphosphonates continuously increase their bone mineral density. This increase is explained in the first 12-18 months following the treatment by the filling of the transient remodeling deficit. Recently, results of a clinical study of alendronate treatment over 7 years still show a continuous increase of bone mineral density. These results raised several questions regarding our understanding of bisphosphonates mode of action.

Methods. Bone remodeling is influenced by different factors including mechanical forces. In the present study, we propose then to consider the effect of bisphosphonates also under biomechanical considerations.

Results. Identification of the model with the clinical data showed that daily treatment of 10 and 20 mg alendronate decreased the bone turnover rate by $2 \%$ and $11 \%$, respectively, in comparison with the $5 \mathrm{mg}$ alendronate treatment. Moreover, the alendronate treatments decreases the resorption threshold stimulus by $19 \%(25 \%, 28 \%)$ for the $5 \mathrm{mg}$ (10 and $20 \mathrm{mg}$, respectively) compared to placebo.

Conclusions. The increase of bone mineral density following bisphosphonates treatment may then be explained by biomechanical considerations. Based on this description, bisphosphonates treatment may indeed change the susceptibility of bone to its biomechanical environment decreasing the mechanical threshold where bone should undergo resorption.
\end{abstract}

\section{Relevance}

This model makes now possible to incorporate the effect of the alendronate in finite element method studies of bone remodeling allowing us to quantify the effect of systemic alendronate treatment following a total hip replacement.

(C) 2003 Elsevier Ltd. All rights reserved.

Keywords: Bone remodeling model; Bisphosphonates; Biomechanics; BMD

\section{Introduction}

In osteoporosis, bone resorption surpasses bone formation activity resulting in bone loss (Boivin et al., 2000). A rational approach to osteoporosis prevention is therefore the use of drugs reducing bone resorption and bone turnover. The underlying hypothesis is that inhi-

\footnotetext{
${ }^{*}$ Corresponding author.

E-mail address: dominique.pioletti@epfl.ch (D.P. Pioletti).
}

bition of bone resorption should enable bone formation to restore bone mass to its homeostasis level, where it should stabilize (Rodan, 1997). It should be kept in mind however, that bone mass is also controlled by mechanical forces.

Bisphosphonates such as alendronate received daily have been shown to continuously increase patients bone mineral density (Liberman et al., 1995) and to preserve the biomechanical properties of the bone (Chavassieux et al., 1997). At the cellular level, bisphosphonates inactivate osteoclasts, which then undergo apoptosis, 
resulting in reduced bone resorption, lower bone turnover, and a positive bone balance (Fleisch, 1996). It has been hypothesized that the reduced bone turnover increases the life span of bone structural units and leads to a more mature bone in which most units approach at least a normal level in their degree of mineralization (Meunier and Boivin, 1997). The lower bone turnover allows the secondary mineralization to be achieved and maintained resulting in an increased bone mineral density (BMD) (Boivin and Meunier, 1999). Based on these observations, BMD increase in the first 12-18 months of antiresorptive drug treatment is explained by the filling of the transient remodeling deficit produced by the time delay between completion of bone resorption and completion of bone matrix formation with its mineralization. Recently, it has been shown that alendronate given without interruption for 7 years still increased lumbar spine BMD (Tonino et al., 2000). This result raises several questions regarding our understanding in BMD increase following antiresorptive agents treatment as the argument for the filling of the transient remodeling deficit is no more a satisfactory explanation for such a long treatment. An alternative explanation to the BMD increase in long term bisphosphonates treatment may be possible by taking into account biomechanical considerations of bone remodeling.

Bone remodeling is inevitably linked to its biomechanical environment. In the present study, a model of bone remodeling including simultaneously the drug and biomechanical effects is then developed. Based on this model, it is hypothesized that BMD increase following bisphosphonates treatments may be explained by the influence of the drug on the bone remodeling parameters. If verified, this hypothesis would support the idea that bisphosphonates treatment may indeed change the susceptibility of bone to its biomechanical environment and would furnish a method of quantifying the drug effect on the bone remodeling. To test this hypothesis, the parameters of the bone remodeling model are identified with published data of alendronate phase III treatment (Liberman et al., 1995).

\section{Methods}

\subsection{Biomechanical bone remodeling model}

To relate bone adaptation to the mechanical stress environment, it has been proposed to link the BMD evolution $\frac{\mathrm{d} \phi}{\mathrm{d} t}$ to its mechanical stimulus $\psi$ by a piece wise linear evolution relation (Fig. 1) (Carter, 1984; Huiskes et al., 1987; Terrier et al., 1997). A lazy zone, where bone neither resorbs nor densifies is delimited by two threshold stimuli $\psi_{\mathrm{r}}$ and $\psi_{\mathrm{d}} \cdot v_{\mathrm{r}}$ and $v_{\mathrm{d}}$ are respectively the bone turnover rate in the resorption and densification regime. It should be noted that the value for the bone turnover

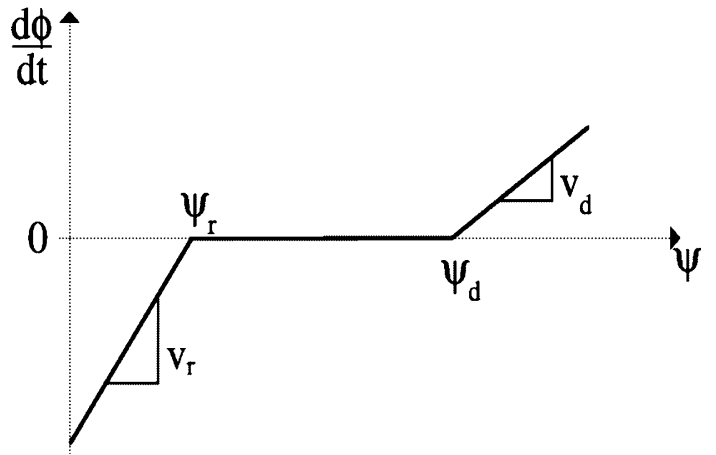

Fig. 1. BMD evolution in function of the mechanical stimuli.

rate is different for the resorption and the densification regime of the bone. The equation describing the bone adaptation based on the piece wise linear evolution can be generally expressed by (Terrier, 1999):

$\frac{\mathrm{d} \phi}{\mathrm{d} t}= \begin{cases}v_{\mathrm{r}}\left(\psi-\psi_{\mathrm{r}}\right) & \psi<\psi_{\mathrm{r}} \\ 0 & \psi_{\mathrm{r}} \leqslant \psi \leqslant \psi_{\mathrm{d}} \\ v_{\mathrm{d}}\left(\psi-\psi_{\mathrm{d}}\right) & \psi>\psi_{\mathrm{d}}\end{cases}$

\subsection{Model of bisphosphonates effect on bone remodeling}

Bisphosphonates such as alendronate mainly affect the resorption process of bone turnover (Chavassieux et al., 1997; Fleisch, 1996). We propose then to model the effects of bisphosphonates by affecting the values of the resorption parameters $v_{\mathrm{r}}$ and $\psi_{\mathrm{r}}$, while the formation parameters $v_{\mathrm{d}}$ and $\psi_{\mathrm{d}}$ are kept constant.

\subsection{Determination of bisphosphonates effect on resorption parameters}

To quantify the bisphosphonates effect on the resorption parameters $v_{\mathrm{r}}$ and $\psi_{\mathrm{r}}$, we used published data of an alendronate phase III treatment (Liberman et al., 1995). In this clinical trial, daily alendronate doses (placebo, 5, 10 , and $20 \mathrm{mg}$ ) were orally given for two years to 994 postmenopausal women presenting osteoporosis. Mean percentage changes in BMD measured at different sites were reported at regular intervals $(0,3,6,12,18$, and 24 months) and showed a continuous increase in the alendronate groups correlated with the doses used.

The measured BMD can be broken down into the contribution of the BMD due to bone formation minus the BMD due to bone resorption. In case of alendronate treatments (and under the assumption of a constant biomechanical stimulus), bone formation should be constant between all groups accordingly to the previously mentioned fact that bisphosphonates does not influence the bone formation. The differences in the measured BMD between groups could then only be explained by a modification of the bone resorption. We used the measured BMD to determine the relative 
difference of the resorption parameters $v_{\mathrm{r}}$ and $\psi_{\mathrm{r}}$ between the different treated groups. The values of the measured BMD at the lumbar spine were used as standard errors were the lowest at this site.

In order to identify the resorption parameters, we write Eq. (1) for the resorption situation. BMD evolution is then given by the following differential equation, which can be used to fit the clinical data (Terrier et al., 1997):

$$
\left\{\begin{array}{l}
\frac{\mathrm{d} \phi}{\mathrm{d} t} \phi(t)^{4}+B \phi(t)^{4}-A=0 \\
\phi\left(t_{0}\right)=\phi_{0}
\end{array}\right.
$$

with $\phi_{0}$ the BMD at time 0 and

$$
\left\{\begin{array}{l}
A=v_{\mathrm{r}} \psi \\
B=v_{\mathrm{r}} \psi_{\mathrm{r}}
\end{array}\right.
$$

Numerical methods are used to solve Eq. (2) from time $t_{0}=0$ to time $t=24$ months in order to determine the $A$ and $B$ values for the placebo and the treated groups. An error function was then defined as the quadratic sum of the difference between the simulated BMD and measured BMD:

$\operatorname{error}(A, B)=\sum_{\alpha=1}^{6}\left[\phi^{\mathrm{m}}\left(t_{\alpha}\right)-\phi^{\mathrm{s}}\left(t_{\alpha}\right)\right]^{2}$

where $\phi^{\mathrm{m}}\left(t_{\alpha}\right)$ is the measured BMD and $\phi^{\mathrm{s}}\left(t_{\alpha}\right)$ is the simulated BMD that depends on the choice of the $A$ and $B$ parameters. Here $t_{\alpha}=0,3,6,12,18,24$ months for $\alpha=1, \ldots, 6$. The FindMinimum function of Mathematica $^{\mathrm{TM}}$ (Wolfram Research, Inc., Champaign, IL, USA) was used in order to find the minimum value of the error function and therefore to find the value of the two parameters $(A$ and $B$ ) that produce the best fit between the simulated and measured BMD. This approach allows us to verify if the increase of BMD in function of bisphosphonates treatment may be explained by biomechanical considerations.

It can be reasonably assumed that the mechanical stimulus (which is a combination of physical activity and body weight) did not significantly differ between groups. The ratios of

$\left(\frac{B_{5 \mathrm{mg}, 10 \mathrm{mg}, 20 \mathrm{mg}}}{A_{5 \mathrm{mg}, 10 \mathrm{mg}, 20 \mathrm{mg}}}\right)\left(\frac{A_{\text {placebo }}}{B_{\text {placebo }}}\right)$

represent indeed the ratios of

$\frac{\psi_{r-5 \mathrm{mg}, 10 \mathrm{mg}, 20 \mathrm{mg}}}{\psi_{r \text {-placebo }}}$.

As mentioned, the rate of bone turnover is different in the resorption and formation regime. The clinical data of the alendronate phase III treatment can then be used to quantify relative bone turnover rate within the alendronate treatment. The ratios
$\frac{A_{10 \mathrm{mg}, 20 \mathrm{mg}}}{A_{5 \mathrm{mg}}}$

represent indeed the ratios of

$\frac{v_{r-10 \mathrm{mg}, 20 \mathrm{mg}}}{v_{r-5 \mathrm{mg}}}$

Calculation of these ratios allowed us to quantify the doses effect of alendronate on the resorption parameters $v_{\mathrm{r}}$ and $\psi_{\mathrm{r}}$.

\section{Results}

Comparison of Eq. (2) with the measured BMD are presented in Fig. 2. The simulated BMD closely fits the measured BMD. The error function values representing the quadratic sum of the difference between the simulated BMD and measured BMD for the placebo and alendronate groups are reported in Table 1. Simulated placebo BMD are quantitatively the closest to the measured BMD, followed by the $5 \mathrm{mg}, 20 \mathrm{mg}$ and finally 10 $\mathrm{mg}$ alendronate groups. Ratios between treatments

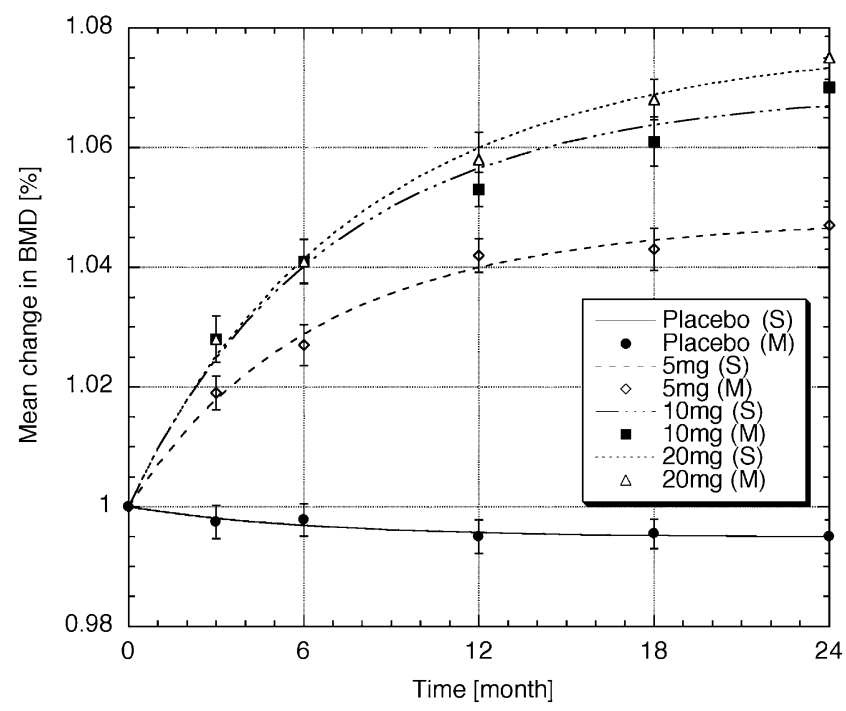

Fig. 2. Mean $( \pm \mathrm{SE})$ changes in bone mineral density of lumbar spine from clinical study $(M)$ (Liberman et al., 1995) and from simulation $(S)$ with the biomechanical model presented.

Table 1

Error function values, ratios of $\psi_{r-x \mathrm{mg}} / \psi_{r \text {-placebo }}$ and $v_{r-x \mathrm{mg}} / v_{r-5 \mathrm{mg}}$ in function of alendronate daily dose

\begin{tabular}{llll}
\hline & Error $(A, B)$ & $\frac{\psi_{r-x \mathrm{mg}}}{\psi_{r \text {-placebo }}}$ & $\frac{v_{r-\mathrm{mmg}}}{v_{r-5 \mathrm{mg}}}$ \\
\hline Placebo & $1.7 \times 10^{-6}$ & - & - \\
$5 \mathrm{mg}$ & $11.2 \times 10^{-6}$ & 0.81 & - \\
$10 \mathrm{mg}$ & $46.8 \times 10^{-6}$ & 0.75 & 0.98 \\
$20 \mathrm{mg}$ & $15.8 \times 10^{-6}$ & 0.72 & 0.89 \\
\hline
\end{tabular}


groups for $\psi_{\mathrm{r}}$ and $v_{\mathrm{r}}$ are reported in Table 1 . The daily treatment with $5 \mathrm{mg}$ alendronate (10 and $20 \mathrm{mg}$, respectively) decrease the resorption threshold stimulus $\left(\psi_{\mathrm{r}}\right)$ by $19 \%(25 \%, 28 \%$, respectively) compared to placebo. The bone turnover rate $\left(v_{\mathrm{r}}\right)$ was decreased by $2 \%$, and $11 \%$, for the 10 and $20 \mathrm{mg}$ treatments respectively compared to the $5 \mathrm{mg}$ treatment.

\section{Discussion}

Bone remodeling or bone mass are controlled by different factors including mechanical forces. The description of the drugs effect on bone remodeling, as bisphosphonates, should then be included in a biomechanical context. Indeed, as noted by Rodan (Rodan, 1997), bone mass changes in response to inhibitors of bone resorption would depend on mechanical function. We developed a model in which the effect of a drug affecting bone remodeling can be accounted for in a general biomechanical approach.

The major result of this study is to show that the alendronate treatment decreases the value of the resorption threshold stimulus $\psi_{\mathrm{r}}$ compared to placebo in a dose dependant relationship. The effect of alendronate tends then to increase the lazy zone where no bone remodeling occurs. An extended lazy zone in the resorption part will then decrease the amount of bone undergoing a resorption process. At a similar level of mechanical stimulus, alendronate treatment will shift the bone balance to an increase bone formation compared to the situation before treatment.

The originality of this study is to quantify the effect of the drugs on bone remodeling with figures. New ways of investigation may then be open to understand the effect of drug affecting bone remodeling. It would be possible to incorporate the effect of alendronate in finite elements method studies of bone remodeling allowing us for example, to quantify the effect of systemic alendronate treatment following a total hip replacement. In this situation, both mechanical (e.g. stress shielding effect) and chemical (drug effect) parameters have to be accounted for simultaneously. Moreover, with the proposed model, it would be possible to compare from a quantitative point of view the effect of different drugs on bone remodeling and to establish a numerical comparison.

In this study, it has been assumed that alendronate treatments had no effect on the bone formation parameters. Experimentally, it has been observed that beside resorption, formation may also decrease when bisphosphonates was used (Rodan and Fleisch, 1996). Nevertheless, no evidence for reduced osteoblastic activity at individual bone formation sites was found. Specifically, effect of alendronate on bone formation has not been observed (Chavassieux et al., 1997). Moreover no adverse effects on bone structure or mineralization was observed, alendronate preserved the biomechanical properties of the bone.

Few studies have tried to theoretically evaluate the effect of drugs on bone remodeling. A mathematical model has shown that the fluoride uptake by skeleton was associated with bone remodeling (Turner et al., 1993). Sensitivity analysis of a bone turnover computer model showed that, in case of antiresorptive drugs, maximal effects on bone volume may be achieved by pharmacologically reducing the activation frequency of bone remodeling units (Lacy et al., 1994). Recently, a study using a computer simulation of bone remodeling evaluated the effect of alendronate treatment (Heaney et al., 1997). It has been found that improvement of bone density by alendronate treatment could be explained by combinations of suppression of bone remodeling activation and positive remodeling balance. The influences of focal bone balance and ash fraction during alendronate treatment have also been compared using a computer simulation of bone remodeling (Hernandez et al., 2001). However, none of these previous studies allowed to incorporate simultaneously in one framework biomechanical and drug effects. The present study was specifically developed for this purpose. It is important to combine these two aspects as mechanical forces are inevitably present in every daily activities.

A major advantage of the proposed model is that the description is not related to a particular mechanical stimulus. Based on the generally accepted concept of bone remodeling using the lazy zone approach (Cowin, 1987; Frost, 1983), several mechanical stimuli have been developed to model the bone remodeling (Huiskes et al., 1987; Terrier et al., 1997). The present description of the alendronate effect is general enough to be used in different proposed models based on the lazy zone concept of bone remodeling. Moreover, the developed approach could be generalized to other drugs affecting bone remodeling. For example, other bisphosphonates such as zoledronate and pamidronate (Pataki et al., 1997), different treatments inhibiting bone resorption such as calcitonin (Rodan and Martin, 2000) or recently interferon- $\beta$ (Takayanagi et al., 2002), or even treatment favoring bone formation such as BMPs (Service, 2000) could be evaluated with the developed model if the BMD evolution is known. A numerical description of different drug effects on bone remodeling could then be performed.

In conclusion, we showed that continuous BMD increase following bisphosphonates treatment may be explained by biomechanical considerations. Bisphosphonates may increase the equilibrium bone remodeling zone on the resorption side in a dose dependent relationship in the developed model. Based on this description, bisphosphonates treatment may indeed change the susceptibility of bone to its biomechanical environment decreasing the mechanical threshold where bone should undergo resorption. 


\section{Acknowledgement}

This work was supported by a grant from the Leenaards Foundation (\#309).

\section{References}

Boivin, G., Meunier, P.J., 1999. How do bone resorption inhibitors increase bone mineral density? Rev. Rhum. Engl. Ed. 66 (11), 534 537.

Boivin, G.Y., Chavassieux, P.M., Santora, A.C., Yates, J., Meunier, P.J., 2000. Alendronate increases bone strength by increasing the mean degree of mineralization of bone tissue in osteoporotic women. Bone 27 (5), 687-694.

Carter, D.R., 1984. Mechanical loading histories and cortical bone remodeling. Calcif. Tissue Int. 36 (Suppl. 1), S19-S24.

Chavassieux, P.M., Arlot, M.E., Reda, C., Wei, L., Yates, A.J., Meunier, P.J., 1997. Histomorphometric assessment of the longterm effects of alendronate on bone quality and remodeling in patients with osteoporosis. J. Clin. Invest. 100 (6), 1475-1480.

Cowin, S.C., 1987. Bone remodeling of diaphyseal surfaces by torsional loads: theoretical predictions. J. Biomech. 20 (11-12), $1111-1120$

Fleisch, H., 1996. The bisphosphonate ibandronate, given daily as well as discontinuously, decreases bone resorption and increases calcium retention as assessed by $45 \mathrm{Ca}$ kinetics in the intact rat. Osteoporos Int. 6 (2), 166-170.

Frost, H.M., 1983. A determinant of bone architecture. The minimum effective strain. Clin. Orthop. (175), 286-292.

Heaney, R.P., Yates, A.J., Santora II, A.C., 1997. Bisphosphonate effects and the bone remodeling transient. J. Bone Miner. Res. 12 (8), 1143-1151.

Hernandez, C.J., Beaupre, G.S., Marcus, R., Carter, D.R., 2001. A theoretical analysis of the contributions of remodeling space, mineralization, and bone balance to changes in bone mineral density during alendronate treatment. Bone 29 (6), 511-516.

Huiskes, R., Weinans, H., Grootenboer, H.J., Dalstra, M., Fudala, B., Slooff, T.J., 1987. Adaptive bone-remodeling theory applied to prosthetic-design analysis. J. Biomech. 20 (11-12), 1135-1150.

Lacy, M.E., Bevan, J.A., Boyce, R.W., Geddes, A.D., 1994. Antiresorptive drugs and trabecular bone turnover: validation and testing of a computer model. Calcif. Tissue Int. 54 (3), 179185.

Liberman, U.A., Weiss, S.R., Broil, J., Minne, H.W., Quan, H., Bell, N.H., Rodriguez-Portales, J., Downs Jr., R.W., Dequeker, J., Favus, M., 1995. Effect of oral alendronate on bone mineral density and the incidence of fractures in postmenopausal osteoporosis. The alendronate phase III osteoporosis treatment study group. N. Engl. J. Med. 333 (22), 1437-1443.

Meunier, P.J., Boivin, G., 1997. Bone mineral density reflects bone mass but also the degree of mineralization of bone: therapeutic implications. Bone 21 (5), 373-377.

Pataki, A., Muller, K., Green, J.R., Ma, Y.F., Li, Q.N., Jee, W.S., 1997. Effects of short-term treatment with the bisphosphonates zoledronate and pamidronate on rat bone: a comparative histomorphometric study on the cancellous bone formed before, during, and after treatment. Anat. Rec. 249 (4), 458-468.

Rodan, G.A., 1997. Bone mass homeostasis and bisphosphonate action. Bone 20 (1), 1-4.

Rodan, G.A., Fleisch, H.A., 1996. Bisphosphonates: mechanisms of action. J. Clin. Invest. 97 (12), 2692-2696.

Rodan, G.A., Martin, T.J., 2000. Therapeutic approaches to bone diseases. Science 289 (5484), 1508-1514.

Service, R.F., 2000. Tissue engineers build new bone [news]. Science 289 (5484), 1498-1500.

Takayanagi, H., Kim, S., Matsuo, K., Suzuki, H., Suzuki, T., Sato, K., Yokochi, T., Oda, H., Nakamura, K., Ida, N., et al., 2002. RANKL maintains bone homeostasis through c-Fos-dependent induction of interferon-beta. Nature 416 (6882), 744-749.

Terrier, A., 1999. Adaptation of bone to mechanical stress: theoretical model, experimental identification and orthopaedic applications [Ph.D.]. EPFL, Lausanne, 1999, p. 175

Terrier, A., Rakotomanana, R.L., Ramaniraka, A.N., Leyvraz, P.F., 1997. Adaptation models of anisotropic bone. Comput. Methods Biomech. Biomed. Eng. (1), 47-59.

Tonino, R.P., Meunier, P.J., Emkey, R., Rodriguez-Portales, J.A., Menkes, C.J., Wasnich, R.D., Bone, H.G., Santora, A.C., Wu, M., Desai, R., et al., 2000. Skeletal benefits of alendronate: 7-year treatment of postmenopausal osteoporotic women. Phase III Osteoporosis Treatment Study Group. J. Clin. Endocrinol. Metab. 85 (9), 3109-3115.

Turner, C.H., Boivin, G., Meunier, P.J., 1993. A mathematical model for fluoride uptake by the skeleton. Calcif. Tissue Int. 52 (2), 130 138. 